\title{
Infection dynamics, dispersal, and adaptation: understanding the lack of recovery in a remnant frog population following a disease outbreak
}

\author{
Donald T. McKnight $\mathbb{D}^{1,2} \cdot$ Leah J. Carr ${ }^{1} \cdot$ Deborah S. Bower $\mathbb{D}^{1,2} \cdot$ Lin Schwarzkopf $^{1} \cdot$ Ross A. Alford ${ }^{1} \cdot$ \\ Kyall R. Zenger ${ }^{1}$
}

Received: 15 February 2020 / Revised: 15 May 2020 / Accepted: 19 May 2020 / Published online: 1 June 2020

(c) The Author(s), under exclusive licence to The Genetics Society 2020

\begin{abstract}
Emerging infectious diseases can cause dramatic declines in wildlife populations. Sometimes, these declines are followed by recovery, but many populations do not recover. Studying differential recovery patterns may yield important information for managing disease-afflicted populations and facilitating population recoveries. In the late 1980s, a chytridiomycosis outbreak caused multiple frog species in Australia's Wet Tropics to decline. Populations of some species (e.g., Litoria nannotis) subsequently recovered, while others (e.g., Litoria dayi) did not. We examined the population genetics and current infection status of $L$. dayi, to test several hypotheses regarding the failure of its populations to recover: (1) a lack of individual dispersal abilities has prevented recolonization of previously occupied locations, (2) a loss of genetic variation has resulted in limited adaptive potential, and (3) $L$. dayi is currently adapting to chytridiomycosis. We found moderate-to-high levels of gene flow and diversity (Fst range: $<0.01-0.15$; minor allele frequency (MAF): 0.192-0.245), which were similar to previously published levels for recovered L. nannotis populations. This suggests that dispersal ability and genetic diversity do not limit the ability of $L$. dayi to recolonize upland sites. Further, infection intensity and prevalence increased with elevation, suggesting that chytridiomycosis is still limiting the elevational range of $L$. dayi. Outlier tests comparing infected and uninfected individuals consistently identified 18 markers as putatively under selection, and several of those markers matched genes that were previously implicated in infection. This suggests that $L$. dayi has genetic variation for genes that affect infection dynamics and may be undergoing adaptation.
\end{abstract}

\section{Introduction}

Recent decades have seen a dramatic increase in emerging infectious diseases in wildlife. These diseases are caused by a diverse range of pathogens (including bacteria, fungi, and viruses), and have afflicted many taxa, often causing devastating declines or even extinctions (Daszak et al. 2000;

Supplementary information The online version of this article (https:// doi.org/10.1038/s41437-020-0324-x) contains supplementary material, which is available to authorized users.

Donald T. McKnight

donald.mcknight@my.jcu.edu.au

1 College of Science and Engineering, James Cook University, Townsville, QLD, Australia

2 School of Environmental and Rural Science, University of New England, Armidale, NSW, Australia
Smith et al. 2006; Scheele et al. 2019). Diseases often shift from being epizootic to being enzootic, and in some cases, populations may rebound following an initial outbreak (Woodworth et al. 2005; McKnight et al. 2017a; Scheele et al. 2017). These recoveries are not guaranteed, and in any one area, some species may recover, while others continue to either decline or persist only in low numbers (McKnight et al. 2017a; Bell et al. 2020). These differential recovery patterns among populations or species may hold important clues for understanding how wildlife populations respond to diseases, and that understanding may enhance conservation efforts and prevent or limit declines in other populations and species.

Chytridiomycosis presents a useful model to study differential recoveries. This disease is caused primarily by the fungal pathogen Batrachochytrium dendrobatidis $(B d)$, and has caused declines and extinctions in hundreds of amphibian species around the world (Berger et al. 1998; Daszak et al. 1999; Lips et al. 2006; Scheele et al. 2019). 


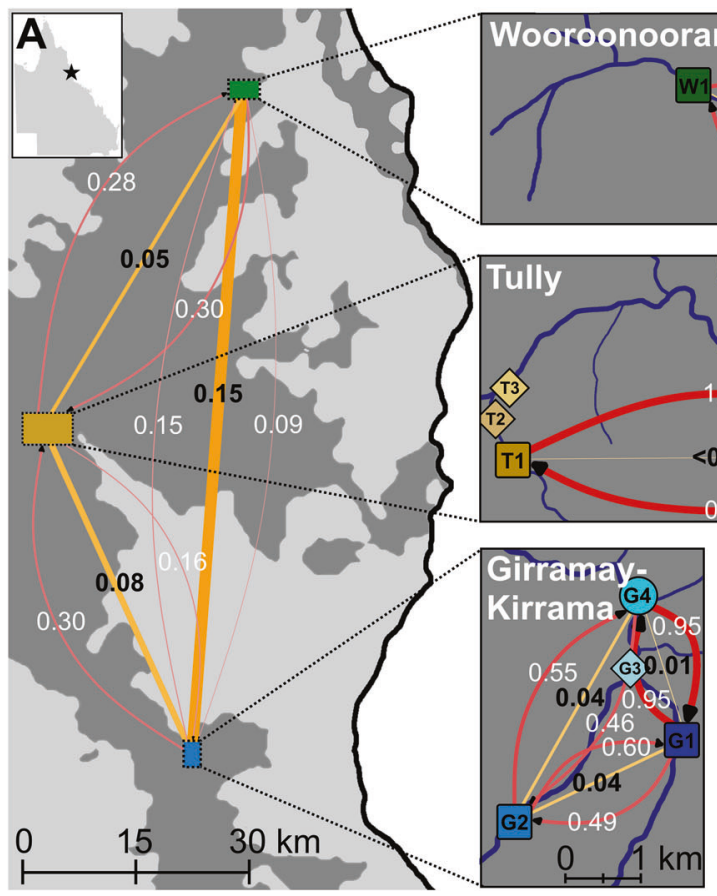

Fig. 1 Study sites and connectivity. a Maps of study sites (symbols and colors correspond to $\mathbf{b}$ and $\mathbf{c}$ ). Large squares $=$ the highest elevation sites (sampled for genetics and $B d$ ), large circles $=$ the lowest elevation sites (sampled for genetics and $B d$ ), and small diamonds = mid-elevation sites (sampled for $B d$ only). Dark-gray areas = rainforest, blue lines $=$ streams, bold black numbers and orange lines $=$ $F s t$ values (the thickness and darkness of the lines are scaled with the $F s t$ ), and white numbers and red lines = relative migration rates from divMigrate (arrows [black] indicate the direction of gene flow; all

The Wet Tropics of Queensland, Australia, experienced a large $B d$ outbreak in the late 1980s and early 1990s, during which several species declined, including greeneyed treefrogs (Litoria serrata [previously genimaculata]), waterfall frogs (Litoria nannotis), and Australian lace-lid frogs (Litoria [previously Nyctimystes] dayi; Ingram and McDonald 1993; Richards et al. 1993; Laurance et al. 1996; McDonald and Alford 1999). Historically, all three species occurred along rainforest creeks at most elevations; however, during the $B d$ outbreak, populations above 300-400-m elevation (hereafter referred to as "upland") either declined sharply ( $L$. serrata) or disappeared (L. nannotis and $L$. dayi), while lowland populations (<300-400 m) remained stable (Richards et al. 1993; Laurance et al. 1996; McDonald and Alford 1999). Following this initial decline, upland $L$. serrata populations quickly recovered at most sites, while L. nannotis gradually recolonized many, but not all, upland sites (McKnight et al. 2017a; Bell et al. 2020). Both of these species now have breeding populations at the headwaters of many upland creeks, despite the fact that $B d$ is still present and continues to infect both species (Richards and Alford 2005; McKnight et al. 2017a). In contrast, L. dayi has not recolonized upland sites, and continues to be restricted to low elevations (McKnight et al. 2017a; Bell et al. 2020).

In a previous study (McKnight et al. 2019), we examined the population genetics of $L$. serrata and $L$. nannotis, and found that both species have high levels of gene flow among populations, but recovered upland populations have reduced genetic diversity. We also found that large areas of high-quality lowland habitat appeared to be important refugia for maintaining diversity during the outbreak. However, the question of why $L$. dayi has not recolonized the upland sites is yet to be addressed. Therefore, in the current study, we built on our previous results by studying the current infection status and population genetics of $L$. dayi. We then compared our results for $L$. dayi with our previously published results on other species.

Specifically, we tested three hypotheses regarding the lack of upland recolonization in L. dayi: (1) Litoria dayi is restricted by low-dispersal ability, which has prevented it from recolonizing upland sites (null hypothesis: $L$. dayi has moderate or high dispersal ability, similar to other species in the area that have recovered from the outbreak), (2) the chytridiomycosis outbreak resulted in a genetic bottleneck 
Table 1 Study site details.

\begin{tabular}{lllllll}
\hline Park & Site & Latitude & Longitude & $\begin{array}{l}\text { Elevation } \\
\text { mean }(\mathrm{m})\end{array}$ & $\begin{array}{l}\text { Elevation } \\
\text { range }(\mathrm{m})\end{array}$ & $\begin{array}{l}\text { Transect } \\
\text { length }(\mathrm{m})\end{array}$ \\
\hline Girramay-Kirrama & G1 & -18.17451 & 145.82828 & 303 & $283-327$ & 610 \\
Girramay-Kirrama & G2 & -18.18250 & 145.80926 & 303 & $287-336$ & 560 \\
Girramay-Kirrama & G3 & -18.16582 & 145.82360 & 222 & $214-225$ & 310 \\
Girramay-Kirrama & G4 & -18.15697 & 145.82381 & 191 & $159-213$ & 1540 \\
Tully & T1 & -17.77420 & 145.59390 & 378 & $359-423^{\mathrm{a}}$ & $350^{\mathrm{a}}$ \\
Tully & T2 & -17.76977 & 145.58993 & 275 & $258-286$ & 220 \\
Tully & T3 & -17.76487 & 145.59011 & 198 & $195-207$ & 330 \\
Tully & T4 & -17.77607 & 145.66484 & 95 & $91-100$ & 150 \\
Wooroonooran & W1 & -17.38523 & 145.86868 & 319 & $295-356^{\mathrm{b}}$ & $560^{\mathrm{b}}$ \\
Wooroonooran & W2 & -17.38814 & 145.87605 & 237 & $224-247$ & 250 \\
Wooroonooran & W3 & -17.39380 & 145.88595 & 134 & $126-143$ & 200 \\
Wooroonooran & W4 & -17.39803 & 145.89468 & 57 & $47-64$ & 450 \\
\hline
\end{tabular}

The coordinates represent the approximate midpoints of each transect. Litoria dayi was not abundant at Girramay-Kirrama, resulting in long transect distances, particularly at the lowest elevation, where it was clustered around small creeks that fed into the main channel. G1 and G2 correspond roughly to DCl and MR1 (respectively) in McKnight et al. (2019).

${ }^{a}$ Only one frog was found above $398 \mathrm{~m}$ (210-m transect length excluding that frog).

${ }^{b}$ Only one frog was found above $334 \mathrm{~m}$ (363-m transect length excluding that frog).

reducing the genetic diversity required for adapting to the disease (null hypothesis: $L$. dayi has moderate or high genetic diversity, similar to other species in the area that have recovered from the outbreak), and (3) Litoria dayi is currently in the process of adapting to $B d$ (null hypothesis: $L$. dayi does not show evidence of adaptation). These three hypotheses were tested by examining current infection patterns, connectivity, and genetic diversity among regions, populations, and individuals, as well as searching for signatures of selection across the genome. These results were then compared with the previously published genetic patterns we documented for $L$. serrata and $L$. nannotis populations.

\section{Materials and methods}

\section{Study sites and samples}

Toe tips (hereafter referred to as tissue samples) were collected from $L$. dayi populations in three areas: Wooroonooran National Park, Tully Gorge National Park (hereafter "Tully"), and Girramay Range/Kirrama Range National Parks (Fig. 1). Girramay and Kirrama border each other and share contiguous forests and streams; therefore, they will be referred to as a single site: "Girramay-Kirrama." At each park, frogs were sampled for DNA at both the highest $(\sim 300-400 \mathrm{~m})$ and lowest $(\sim 50-200 \mathrm{~m})$ elevations currently occupied by $L$. dayi (Table 1). At Wooroonooran, frogs were sampled at two points along Pugh Creek. At Tully, L. dayi was sampled at
Python Creek and an unnamed creek (both creeks feed into the Tully River), and at Girramay-Kirrama, frogs were sampled from two creeks at the current highest elevation for $L$. dayi (these sites correspond to "DCl" and "MRl" in our previous study on L. nannotis and L. serrata, McKnight et al. 2019). Both creeks connect below those sampling sites, so a third site was sampled downstream, at the lowest elevation for $L$. dayi at Girramay-Kirrama. At all three parks, there was a direct water connection between the highest and lowest elevation sites.

In addition to the tissue samples, we also collected data on the current infection status of $L$. dayi, and examined how elevation influenced infection. The expectation was that if $B d$ is preventing $L$. dayi from recolonizing upland sites (as opposed to a lack of dispersal ability or genetic diversity), then both infection prevalence and intensity should increase with increasing elevation. To test this, skin swabs were collected from all captured frogs at each tissue-sampling site, and frogs were swabbed at additional sites between our tissue-sampling sites (two each at Tully and Wooroonooran, and one at Girramay-Kirrama). These sites were selected to cover the entire current elevation range of $L$. dayi, with roughly evenly spaced sampling points at each park. More details on sampling sites and sample sizes are provided in Fig. 1 and Tables 1 and 2.

At each site, frogs were sampled at night by walking a transect starting at the highest point where $L$. dayi could be found at upland sites, and at the lowest point where $L$. dayi could be found at lowland sites. Every $L$. dayi encountered was sampled until a minimum representative number $(n \sim$ 30) had been reached, or no more $L$. dayi could be found. 
Table 2 Sample sizes for each site.

\begin{tabular}{|c|c|c|c|c|c|c|c|c|c|}
\hline \multirow[t]{2}{*}{ Site } & \multirow[t]{2}{*}{ Tissue } & \multicolumn{4}{|c|}{ All $B d$ swabs } & \multicolumn{4}{|c|}{$\begin{array}{l}B d \text { swabs for tissue } \\
\text { samples }\end{array}$} \\
\hline & & Total & $\begin{array}{l}B d+ \\
(\geq 10)\end{array}$ & $\begin{array}{l}B d+ \\
(<10)\end{array}$ & $B d-$ & Total & $\begin{array}{l}B d+ \\
(\geq 10)\end{array}$ & $\begin{array}{l}B d+ \\
(<10)\end{array}$ & $B d-$ \\
\hline G1 & 28 & 30 & 17 & 6 & 7 & 27 & 15 & 5 & 7 \\
\hline G2 & 19 & 18 & 8 & 8 & 2 & 18 & 8 & 8 & 2 \\
\hline G3 & - & 27 & 11 & 7 & 9 & - & - & - & - \\
\hline G4 & 23 & 28 & 11 & 8 & 9 & 21 & 9 & 5 & 7 \\
\hline $\mathrm{T} 1$ & 28 & 27 & 11 & 7 & 9 & 25 & 11 & 7 & 7 \\
\hline $\mathrm{T} 2$ & - & 27 & 9 & 12 & 6 & - & - & - & - \\
\hline $\mathrm{T} 3$ & - & 28 & 4 & 10 & 14 & - & - & - & - \\
\hline $\mathrm{T} 4$ & 28 & 29 & 4 & 6 & 19 & 26 & 2 & 5 & 19 \\
\hline W1 & 29 & 29 & 21 & 5 & 3 & 28 & 20 & 5 & 3 \\
\hline W2 & - & 29 & 18 & 5 & 6 & - & - & - & - \\
\hline W3 & - & 25 & 11 & 7 & 7 & - & - & - & - \\
\hline W4 & 28 & 29 & 2 & 6 & 21 & 28 & 2 & 5 & 21 \\
\hline
\end{tabular}

Tissue tissue samples sequenced, All Bd swabs all swabs that amplified in all three triplicates, Bd swabs for tissue samples swabs that amplified in all three triplicates, and were from frogs that were sequenced (one frog did not have a swab), $B d+(\geq 10)$ swabs that were $B d$-positive and had $\geq 10$ zoospore equivalents of DNA, $B d+(<10)$ swabs that were $B d$-positive but had $<10$ zoospore equivalents of DNA, $B d-$ swabs that showed no amplification in all three triplicates.

At Girramay-Kirrama, L. dayi was rare, resulting in long transects, particularly at the lowest elevation, whereas at Tully, it was abundant, resulting in short transects (Table 1). Female $L$. dayi spend most of their time in the forest and are seldom found along streams (Hodgkison and Hero 1999). As a result, all samples were collected from males, with the exception of one female at Girramay-Kirrama, one female at Tully (swab only), and one juvenile at Tully. All sampling took place in September 2017, and each site was sampled during a single night or, if necessary, on two consecutive nights. No recaptures occurred when sampling took two nights.

Each frog was captured in a clean plastic bag, handled using a new pair of nitrile gloves, and released at its collection site within minutes of being captured. Tissue samples were collected via toe tips (one from each rear foot). This procedure is minimally invasive and does not typically result in bleeding. The scissors were dipped in ethanol and flame-sterilized between each frog. Tissues were stored in vials of $70 \%$ ethanol. Skin swabs were collected by rubbing a sterile, rayon-tipped swab (Medical Wire, MW113), along the stomach, thighs of each rear leg, and each rear foot (five times each, 25 strokes in total). All samples were kept at room temperature for up to $48 \mathrm{~h}$, after which they were placed on ice for transport. Tissue samples were stored at $4{ }^{\circ} \mathrm{C}$, and $B d$ swabs were stored at $-20{ }^{\circ} \mathrm{C}$.

\section{Bd extraction, $\mathrm{qPCR}$, and analysis}

To assess the infection status of $L$. dayi, the DNeasy Blood and Tissue DNA extraction kit (Qiagen) was used for each skin swab following the standard protocol, with two modifications: an overnight digestion and two $20 \mu \mathrm{l}$ elutions. The extracted DNA was sent to Cesar Australia, a commercial company, to perform triplicate qPCR following the standard protocol outlined in Boyle et al. (2004). Frogs were only considered $B d$-positive if $B d(\geq 0.1$ zoospore equivalent) was identified in all three of the qPCR triplicates. Eighteen samples amplified in only one or two of the triplicates. These 18 samples were considered "ambiguous" and were removed from all analyses. To control for contamination, we made six blanks (i.e., clean swabs that were placed in vials without swabbing frogs) and extracted them, and ran qPCR alongside the actual samples. There was no amplification in any of the blanks. Ceasar Australia also tested a subset of 31 samples for PCR inhibition using the TaqMAN Exogenous Internal Positive Control (VIClabeled) kit, and did not find inhibition in any of the samples.

A binomial generalized linear regression model in $R(R$ Core Development Team 2017) was used to look for an association between $B d$ infection prevalence (i.e., proportion of individuals that were infected) and elevation. Infection status was the response variable, and elevation, park, and their interaction were the explanatory variables. All individuals were included, regardless of infection status or intensity (excluding the 18 ambiguous samples). To test for an association between infection intensity (i.e., zoospore load) and elevation, a linear model in $\mathrm{R}$ was used. Infection intensity ( $\log _{10}$ of qPCR results) was the response variable, and elevation, park, and their interaction were the explanatory variables. All $B d$-positive individuals were included in this model, regardless of zoospore load. The significance of the relationships was tested using the Anova function in the car package (Fox and Weisberg 2011) with a type 2 sum of squares. For the binomial model, overdispersion was checked by dividing the residual deviance by the degrees of freedom (1.17), and for the linear model, a residual and QQ plot were used to assess model fit.

\section{Genomic DNA extraction and sequencing}

Genomic DNA was extracted from each sample using the cetyl trimethyl ammonium bromide procedure (with chloroform precipitation, Doyle and Doyle 1987), and the quality and quantity of DNA were checked using gel electrophoresis and a Nanodrop DNA/RNA spectrophotometer analyzer. Genome-wide single-nucleotide polymorphisms (SNPs) were generated by Diversity Arrays Technology (DArT PL), Canberra, Australia, using their proprietary DArTSeq $^{\mathrm{TM}}$ genotyping by sequence methodology 
(Sansaloni et al. 2011; Kilian et al. 2012). The same approach was previously used to generate the high-quality SNP data for L. serrata and L. nannotis that we used for comparisons (McKnight et al. 2019), and is explained in detail elsewhere (Lal et al. 2017; Lind et al. 2017; Kjeldsen et al. 2019).

Briefly, DArTSeq ${ }^{\mathrm{TM}}$ SNP genotyping used both frequent and rare restriction enzymes, Pst I and $S p h \mathrm{I}$, to perform a joint digestion-ligation reaction at $37^{\circ} \mathrm{C}$ for $2 \mathrm{~h}$ with $\sim 100-200 \mathrm{ng}$ of individual gDNA. Custom proprietary barcoded adapters were used in the ligation reaction to facilitate individual sample recognition, and allow for primer-binding sites. Each sample was individually PCRamplified using custom primers to selectively enrich target fragments for sequencing and was visualized via gel electrophoresis. Samples that displayed incomplete or nonuniform digestion and amplification patterns were removed from the library. A minimum of $15 \%$ random technical replicates were included for downstream quality and performance measurements. Batches of individuals were pooled and sequenced (77 cycles) on a single flow-cell lane on an Illumina HiSeq 2500 according to the manufacturer's recommendations.

Individual raw fastq sequence data were demultiplexed and adapters trimmed with any individual read containing base-pair Q scores < 30 removed. All sequence data were checked against DArTdb database sequences to identify read contamination for removal (Sivasankaran et al. 1993). SNP calling was conducted using the DArTsoft14 software within the KDCompute analyses pipeline developed by Diversity Arrays Technology (http://www.kddart.org/ kdcompute.html) following Lind et al. (2017) and Kjeldsen et al. (2019). KDCompute first created clusters (or stacks) of identical reads at a dataset level with three nucleotide mismatches allowed. These sequence clusters were then compared and matched with one another to identify polymorphisms. Following SNP identification, individual locus performance metrics were generated, including call rate, homozygote and heterozygote proportions, polymorphic information content, allele average read depth, and reproducibility (based on technical replicates).

\section{Post-genotyping filtering and quality control}

DArTSeq sequencing and filtering pipelines delivered a total of 33,016 SNPs. To obtain the highest-quality data, SNPs were further filtered by first removing duplicate SNPs within the same sequence reads (69 base pairs), and sequences with a high degree of similarity (assigned with a 95\% probability, Lal et al. 2017). Next, the following criteria were applied: average number of reads (averaged between the two alleles) $\geq 7, \mathrm{MAF} \geq 0.02$, call rate $=1.0$ (i.e., no missing data), and reproducibility $\geq 0.9$. A very stringent call rate was used because of the possible presence of null alleles at some parks (McKnight et al. 2019).
To identify potential outlier loci under selection, BayeScan v.2.1 (false discovery rate $[$ FDR $]=0.1$, Foll and Gaggiotti 2008; Foll 2012), HacDivSel (Carvajal-Rodriguez 2017), and FstHet (Flanagan and Jones 2017) were used both on the entire dataset (with each collection site as a population) and on each park separately (at Girramay-Kirrama, both higher elevation sites [G1 and G2] were entered as a single population; HacDivSel was not used for the entire dataset because it requires datasets within only two populations). This produced four sets of tests (one for the entire dataset and one for each park). Any markers that were identified as outliers in at least two programs for any of the four sets of tests were removed, producing a neutral dataset. This procedure was used simply for the purpose of making a neutral dataset (neutral among populations), not for identifying markers that were under selection specifically for $B d$.

Once a neutral dataset had been constructed, PLINK (v1.9, Purcell et al. 2007) was used to test for linkage disequilibrium (LD, all individuals were included in the analysis). Any links with an $R^{2} \geq 0.6$ were removed. To minimize the loss of data, this was done by iteratively removing the SNPs with the greatest number of significant links, until no links $\geq 0.6$ remained.

The GWASExactHW package in R (v1.01, Painter 2013) was used to identify markers that were out of Hardy-Weinberg equilibrium (HWE). This test was performed with all the sites within each park combined into a single population. Any markers that were significantly out of HWE $(P<0.01)$ at all populations were removed $(P$ values were not adjusted for multiple comparisons, resulting in the retention of a conservative set of markers).

These filtering steps resulted in a final dataset of 8,304 high-quality, neutral SNPs. With the exception of the call rate threshold and the filtering criteria for neutral markers (i.e., outlier tests), these were the same filtering steps used in McKnight et al. (2019) for L. serrata and L. nannotis.

\section{Population structure and connectivity}

Several methods were used to examine population structure and connectivity, thus testing the hypothesis that $L$. dayi has low physical dispersal abilities that are preventing it from recolonizing the uplands. First, the genetic distances among populations were calculated as $F s t$ values in Arlequin (v3.5.2.2, Excoffier et al. 2005). Second, the divMigrate function in the R package diveRsity (v1.9.90, Keenan et al. 2013) was used to examine differential migration rates.

Population structure was visualized using both NetView R (v1.0, Steinig et al. 2015) and a discriminant analysis of principal components (DAPC) via the $\mathrm{R}$ package "adegenet" (v2.0.1, Jombart 2008, Fig. 1). In addition, an analysis of molecular variance (AMOVA) in Arlequin was used 
to examine how the variance was partitioned among and within parks (parks were included as the groups, with sampling sites within parks included as the populations).

\section{Genetic diversity}

Genetic diversity was examined both within each sampling site and within each park (all sampling sites combined). The following metrics were calculated: MAFs, percent of markers that were polymorphic within a given site or park (both with and without rarefying), expected and observed heterozygosities (Genetix v4.05.2, Belkhir 2004), and Fis (Genetix). In addition, the effective population size $\left(N_{\mathrm{e}}\right)$ was calculated using the LD method in NeEstimator, using only alleles with a MAF $>0.05$ (v2.01, Do et al. 2014).

\section{Adaptation}

To construct a dataset to identify markers under selection for $B d$, the data were carried through the previously described filtering steps (except for removing outliers, SNPs in LD, and those out of HWE) following the same settings used before, with one exception. Only using individuals that were not missing any data resulted in a large loss of markers. Therefore, to maximize the chance of detecting selection, a less-stringent threshold of $70 \%$ (i.e., less than $30 \%$ missing data) was used. Then, to reduce the presence of null alleles, for each SNP, a Fisher's exact test was used to compare the amount of missing data among the three parks, and any SNPs that were significantly different at a FDR of 0.05 were removed.

Outlier tests were conducted in BayeScan $(F D R=0.1)$ (Foll and Gaggiotti 2008; Foll 2012), HacDivSel (CarvajalRodrõ Âguez 2017), and FstHet (Flanagan and Jones 2017). In each test, uninfected individuals were compared with individuals that were infected with a load equivalent to the DNA of at least ten zoospores. This threshold was selected a priori and used to reduce noise in the tests by removing individuals who may have simply carried a few zoospores acquired from the environment without actually being infected.

The fundamental concept behind each outlier detection program is that alleles under selection will differ in frequency between two (or more) groups more strongly than the background difference between the groups (i.e., those markers are "outliers"). In our case, the expectation was that if an allele was under selection due to a role in $B d$ infection dynamics, the difference in the allele frequencies between infected and uninfected individuals would exceed the expected difference based on the background level of differentiation between those groups. Each program applies a different strategy to identify outliers (e.g., BayeScan uses a Bayesian approach based on the multinomial Dirichlet model, whereas FstHet and HacDivSel apply frequentist methods), generally resulting in different outcomes from each method. Some of these results can be spurious for a variety of reasons, such as a mismatch between the evolutionary mechanism that produced the observed differences and the expected mechanism based on a program's assumptions. Therefore, we implemented a conservative approach, and only considered markers as significant outliers (i.e., putatively under selection) if they were identified as outliers in all three tests. This provided a robust set of markers that had a high probability of being under selection.

We could not perform tests on subsets of the data (e.g., for an individual park or elevation) due to small sample sizes for subsets. Therefore, to control for differences among parks and elevations, the genetic distance (1-proportion of shared alleles) among individuals was calculated using only the markers that were identified as outliers in all tests. Then the adonis2 function in the $\mathrm{R}$ package vegan (Oksanen et al. 2017) was used to run a PERMANOVA with elevation and park as the first terms in the model, followed by $B d$, which was included as a binary factor (infected $[\geq 10$ zoospore equivalents] or uninfected [0 zoospore equivalents]). All interactions were included (5,000 iterations). This tested whether infected and uninfected individuals were genetically distinct (based on the markers that were putatively under selection) after accounting for the effects of elevation and park. To verify the utility of the PERMANOVA for this application, the probability that a random subset of markers would return a significant difference between infected and uninfected individuals was assessed by repeatedly $(1,000$ iterations) randomly selecting 18 markers (the number that was significant in all three outlier tests), and re-running the PERMANOVA using only those markers to calculate genetic distance.

In addition, for markers that were identified as outliers in all three tests, an NCBI BLAST (blastn) search was used to align them with the following genomes: Xenopus (taxid: 8353), Rana (taxid: 8399), and Nanorana (taxid: 120497). For each sequence, the best two matches (based on the lowest $e$ value) were retained for further examination. An identical BLAST search was also conducted for four markers that were identified as outliers in both BayeScan (the most conservative method) and FstHet, but not HacDivSel (no markers were identified as outliers in BayeScan and HacDivSel, but not FstHet). NCBI, UniProt, GeneCards, and Xenbase were used to determine the putative functions of the genes.

\section{Results}

\section{Infection status}

Both $B d$ infection intensity $\left(F_{1,208}=8.05, P=0.005\right)$ and prevalence $\left(\chi_{1,320}^{2}, P<0.001\right)$ increased significantly with 
increased elevation (Figs. 2 and 3). There were also significant differences among parks in both analyses $\left(F_{2,208}=\right.$ 6.36, $P<0.001 ; \chi_{2,320}^{2}, P=0.021$, respectively), but interactions were not significant $\left(F_{2,208}=0.54, P=0.586\right.$; $\chi_{2,320}^{2}, P=0.137$, respectively). Infection prevalences at the highest elevations within each park were high, with $66.7-89.7 \%$ of individuals infected with $B d$.

\section{Low-dispersal hypothesis}

There was no evidence of genetic subdivision within parks (all $F s t \leq 0.04$ ) and only moderate differences among parks (all Fst $\leq 0.15$, Fig. 1). Similarly, both NetView (Fig. 1b) and DAPC (Fig. 1c) showed that each park clustered separately from the others, but there was little evidence of substructure within parks (nearly all of the variation in the DAPC was explained by differences among parks, Fig. 1c). Further, the AMOVA found that differences among parks accounted for $9.05 \%$ of the variation in the data, whereas differences among sampling sites within parks only explained $1.55 \%$ of the variation (differences among individuals within sites $=2.11 \%$; variation within individuals
$=87.3 \%)$. The divMigrate results suggested that gene flow was bidirectional along the streams (Fig. 1a).

\section{Loss of genetic diversity hypothesis}

Diversity analyses did not suggest inbreeding or a large loss of diversity (Table 3, Fig. 4). Girramay-Kirrama had slightly reduced diversity compared with the other parks, but expected and observed heterozygosities were similar at all sites, and Fis values did not deviate substantially from zero. Similarly, the average MAFs per site ranged from 0.192 to 0.245 , and the percentage of polymorphic markers within each site ranged from 79.0 to $97.8 \%$. Nevertheless, $N_{\mathrm{e}}$ estimates were low at Girramay-Kirrama (7.9-40.3 per site) and Wooroonooran (38.0-63.3 per site).

\section{Adaptation hypothesis}

BayeScan identified 22 outlier loci, HacDivSel identified 422, and FstHet identified 492 (550 markers in total). Eighteen markers were identified as outliers by all three programs, and four were identified by BayeScan and dendrobatidis infection prevalence at each study site (i.e., percent of individuals that were infected). Sites within parks are arranged from the lowest elevation (G4, T4, and W4) to the highest elevation (G2, G1, T1, and W1). Black dots are the mean elevation for a given site, and the error bars show the range.
Fig. 2 Batrachochytrium
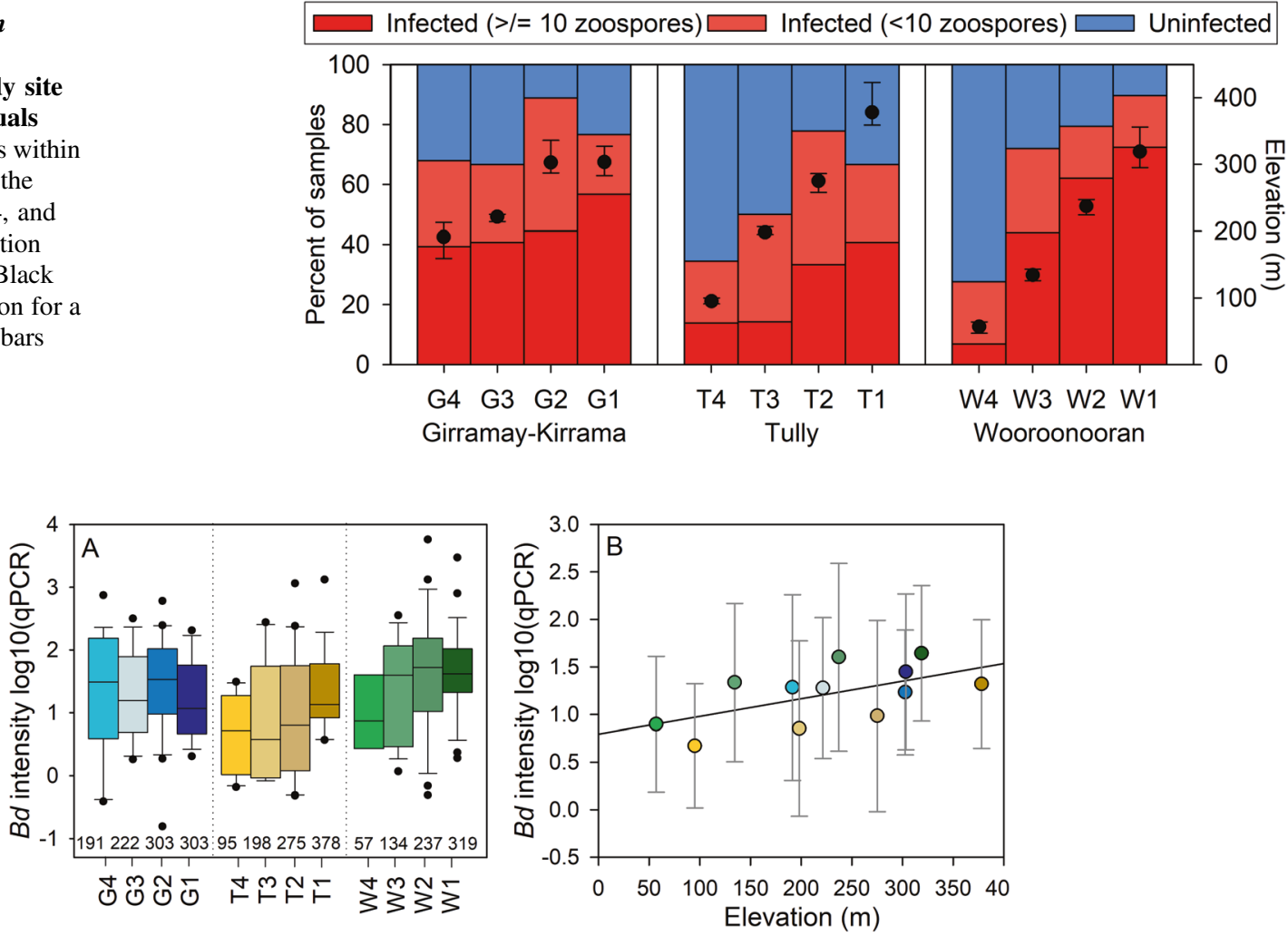

Fig. 3 Batrachochytrium dendrobatidis infection intensity (i.e., $\log _{10}$ of the zoospore load based on the qPCR results for all infected individuals). In both panels, colors are used consistently and correspond to the sites in Fig. 1. a Infection intensity at each sampling site. Numbers show the mean elevation per site. G Girramay-Kirrama, T
Tully, W Wooroonooran. Whiskers show the 10th/90th percentile, and all outliers are shown. b Mean infection intensity for each site plotted against mean elevation for that site. Error bars show one standard deviation for the infection intensity. Colors correspond to sampling sites in Fig. 1a. 
Table 3 Diversity results for each site and for each park (i.e., all sites within a park combined).

\begin{tabular}{lllllllrc}
\hline & Mean MAF (SD) & \% poly. & $\%$ poly. rare & H n.b. & Het. obs. & Fis (SD) & $\begin{array}{l}N_{\mathrm{e}} \text { (jackknife CI) } \\
\text { [NeEstimator] }\end{array}$ \\
\hline G1 & $0.204(0.158)$ & 86.4 & 84.4 & 0.280 & 0.270 & $0.034(0.200)$ & $40.3(22.2-114.7)$ \\
G2 & $0.192(0.162)$ & 79.0 & 79.0 & 0.265 & 0.272 & $-0.021(0.246)$ & $7.9(4.8-12.5)$ \\
G4 & $0.204(0.158)$ & 85.1 & 84.0 & 0.281 & 0.282 & $-0.002(0.218)$ & $22.1(9.9-98.5)$ \\
T1 & $0.243(0.143)$ & 97.5 & 96.3 & 0.333 & 0.323 & $0.033(0.208)$ & $67.7(25.9-\infty)$ \\
T4 & $0.243(0.141)$ & 97.8 & 96.3 & 0.334 & 0.321 & $0.044(0.207)$ & $7499.3(3086.5-\infty)$ \\
W1 & $0.236(0.146)$ & 95.6 & 94.1 & 0.324 & 0.315 & $0.028(0.206)$ & $63.3(34.6-211.6)$ \\
W4 & $0.237(0.146)$ & 95.6 & 93.9 & 0.324 & 0.315 & $0.028(0.210)$ & $38.0(19.1-150.2)$ \\
G & $0.207(0.157)$ & 90.5 & 89.8 & 0.282 & 0.274 & $0.027(0.143)$ & $57.0(40.7-86.1)$ \\
T & $0.245(0.140)$ & 98.9 & 98.9 & 0.334 & 0.322 & $0.042(0.157)$ & $307.4(124.8-\infty)$ \\
W & $0.241(0.143)$ & 97.6 & 97.6 & 0.327 & 0.315 & $0.039(0.158)$ & $87.3(53.5-187)$ \\
\hline
\end{tabular}

$M A F$ minor allele frequency, $\%$ poly. percent of markers that were polymorphic at a given site, \% poly. rare percent of markers that were polymorphic at a given site after rarefying the data to the lowest sample size, $H$ n.b. expected heterozygosity (corrected), Het. obs. observed heterozygosity, Fis (SD) mean inbreeding coefficient and SD of the mean (median values ranged from -0.008 to 0.000 ), $N_{e}$ effective population size (via NeEstimator).

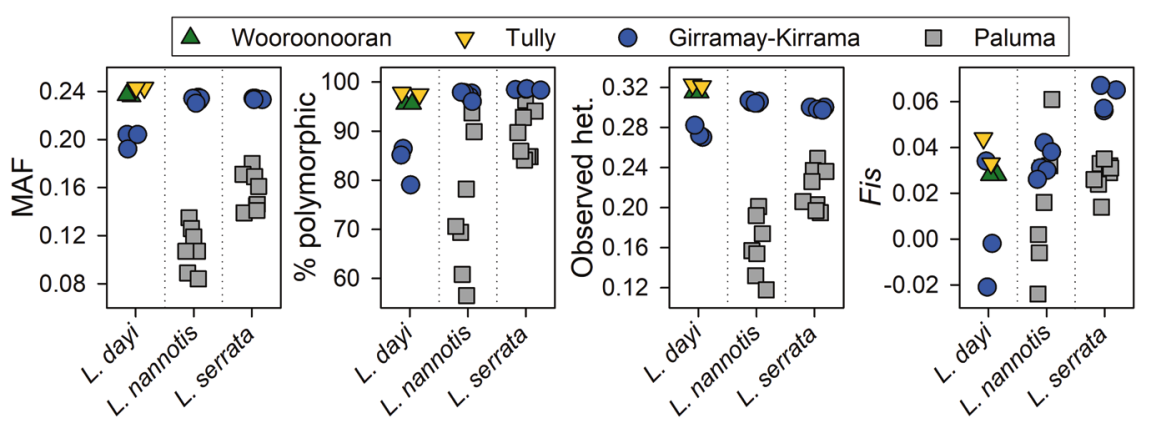

Fig. 4 Genetic diversity metrics from this study (L. dayi) compared with the previously reported results for $L$. serrata and $L$. nannotis (McKnight et al. 2019). Each point is a sampling site. MAF $=$ minor

FstHet, but not HacDivSel (no markers were identified as outliers in BayeScan and HacDivSel, but not FstHet). The PERMANOVA based on the 18 consensus markers found a significant effect of $B d$ (pseudo $P<0.001$ ) after accounting for elevation (pseudo $P<0.001$ ) and park (pseudo $P<0.001$ ); however, elevation and park explained more of the variation (elevation $R^{2}=0.137$, park $R^{2}=$ $0.222, B d R^{2}=0.062$ ). The only significant interaction was between elevation and park (pseudo $P=0.005$; Supplementary Information). For the 1,000 PERMANOVAs using the same model structure and individuals, but random subsets of 18 markers, only $4.2 \%$ showed a significant effect of $B d$ (pseudo $P<0.05$ ), which is close to the expected type 1 error rate. The lowest pseudo $P$ value from random subsets of markers was 0.0026 , whereas our pseudo $P$ value for the 18 consensus markers was 0.0002 . This suggests that it is unlikely that our results arose by chance, and the 18 consensus markers likely constitute an actual genetic difference between infected and uninfected allele frequency, $\%$ polymorphic $=$ percent of markers that were polymorphic in a given site, observed het. = observed heterozygosity.

frogs. This difference can be visualized with ordination plots (Fig. 5).

The BLAST search found matches for all 22 of the potential outliers we tested (18 consensus markers +4 that were found in BayeScan and FstHet but not HacDivSel). We could not find standard gene ontology terms for many of these sequences; therefore, we used the information available in NCBI, UniProt, GeneCards, and Xenbase to assign the sequences into categories of putative functions or systems (see Supplementary Information for details about the SNPs and BLAST results). SNP sequences with the highest BLAST scores (based on $e$ values [0.003-4.9]) were grouped into the following categories based on biological functionality (five fit into two categories): cell surface functions (recognition, movement, and transport across the membrane $=6, \quad$ DNA/RNA (transcription, maintenance, and repair) $=3$, skin $=3$, tumor regulation and apoptosis $=$ 3 , organelle structure and cytoskeleton $=3$, nervous system $=3$, immune system $=1$, nuclear transport $=1$, meiosis $=1$, 


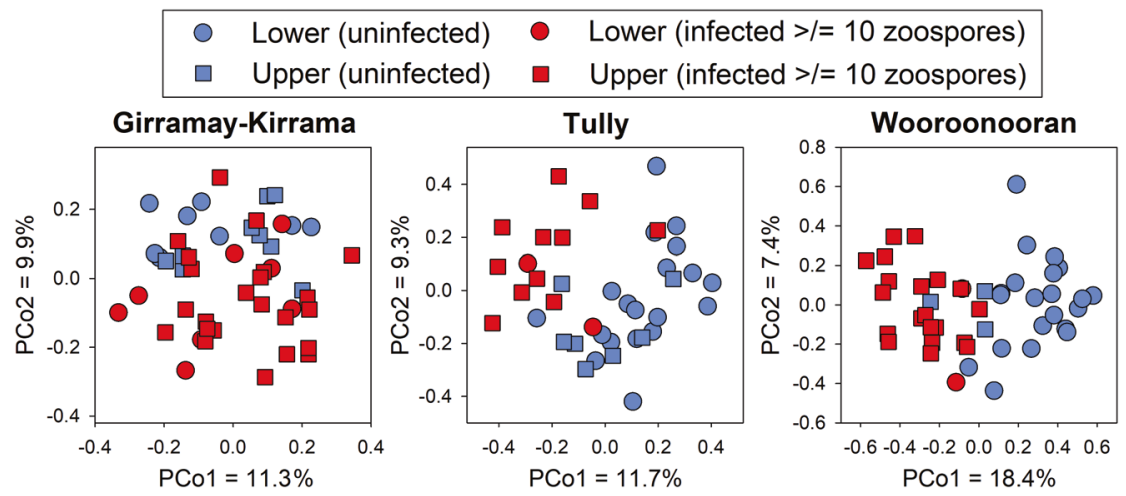

Fig. 5 Ordination plots (PCoA) based on genetic distances (1-proportion of shared alleles) that were calculated using only 18 SNPs that were identified as potentially being under selection in all three outlier detection tests. Lower $=$ the lowest elevation site at each park (G4, T4, and W4), upper $=$ the highest elevation site(s) at each park $(\mathrm{G} 1, \mathrm{G} 2, \mathrm{~T} 1$, and $\mathrm{W} 1)$. Only individuals that were uninfected $(0$ zoospore equivalents) or infected with $\geq 10$ zoospore equivalents were included. and unknown or matches to full sequences rather than genes $=3$. The second-best BLAST matches (based on $e$ values) were similar (20 matches; three fit into two categories): cell surface functions (recognition, movement, and transport across the membrane) $=3$, skin $=3$, tumor regulation and apoptosis $=3$, organelle structure and cytoskeleton $=3$, DNA/RNA (transcription, maintenance, and repair) $=2$, immune system $=1$, nervous system $=1$, GTPase activation $=1$, and unknown or matches to full sequences rather than genes $=6$. Several of these (e.g., genes related to skin function and cell cycle regulation) have obvious implications for $B d$ and are consistent with previous studies. Nevertheless, it should be noted that due to the short length of our sequences, and the lack of genomes for closely related species, many of these matches were low quality (only 10 markers had a match with an $e$ value $<1$, the remaining matches had $e$ values of 1.4 or 4.9).

\section{Discussion}

Our results suggest that $L$. dayi has both high population connectivity (consistent with effective dispersal) and high levels of genetic diversity (comparable with those of $L$. nannotis and $L$. serrata). The results allowed us to test the hypotheses we advanced in the "Introduction" regarding possible effects of dispersal, bottlenecking, selection, and ongoing infection on the lack of recovery in $L$. dayi.

\section{Low-dispersal hypothesis}

Our results are not consistent with the hypothesis that lowdispersal abilities have prevented $L$. dayi from recolonizing upland sites. Population connectivity provides a useful proxy for dispersal ability, and we observed high levels of connectivity within each park (based on Fst values), and both NetView and the DAPC showed little evidence of structuring. In addition, divMigrate did not detect asymmetry in the gene-flow patterns, suggesting that frogs were moving both upstream and downstream. Although a downstream bias in gene flow is common in some streamdwelling species (Bolnick et al. 2008; Guarnizo and Cannatella 2013), its absence in $L$. dayi makes sense, because their eggs are attached to rocks, and their tadpoles possess adaptations to fast-flowing water, such as suctorial mouth disks and specialized tails, to prevent them from being washed downstream (Davies and Richards 1990).

The $F s t$ values for $L$. dayi were similar to the previously reported values for $L$. nannotis (which went through the same pattern of declines, but has recolonized many upland sites). Indeed, at Girramay-Kirrama, where our G1 and G2 sites correspond to L. nannotis sampling sites in McKnight et al. (2019), L. dayi only had a slightly higher $F$ st than L. nannotis (0.04 compared with 0.01), and when looking across similar sites from each study, the ranges of $F s t$ values were similar for both species ( $L$. dayi: $<0.01-0.04$; L. nannotis: $0.01-0.08$; only sites with direct water connections were included in these ranges). These results are also consistent with previous work showing that $L$. dayi moves away from streams, with females spending most of the year in the forest (Hodgkison and Hero 1999).

Taken together, these results do not suggest a lowdispersal ability in $L$. dayi. Indeed, the similarities to previously reported values and patterns for L. nannotis suggest that both species have similar dispersal abilities. Therefore, given that $L$. nannotis experienced the same declines, at the same sites, as $L$. dayi, but has recolonized many upland sites, a lack of dispersal ability in $L$. dayi does not appear to explain its lack of recovery. Another possibility, suggested by Bell et al. (2020), is that $L$. dayi has failed to recolonize 
upland sites because of limited dispersal opportunities due to small lowland population sizes, rather than an inherent lack of dispersal ability. Although this may have played a role at Girramay-Kirrama, L. dayi was abundant in the lowlands at Tully and Wooroonooran, making this hypothesis unlikely at those sites.

In addition, the infection data showed that both the prevalence (percent of individuals infected) and intensity (zoospore load) of infections increased with elevation, with $66.7 \%$ or more of individuals infected at the highest elevations currently occupied by $L$. dayi. This result agrees with an L. dayi survey conducted in 2013 (Bell et al. 2020), and suggests that $B d$ is likely still a substantial problem for $L$. dayi, and continues to restrict its upper elevational range.

\section{Loss of genetic diversity hypothesis}

Litoria dayi had high levels of genetic diversity, and our results do not suggest that a lack of diversity has prevented it from adapting and recovering from the disease outbreak. Although the diversity was slightly lower at Girramay-Kirrama than at Wooroonooran or Tully, possibly as a result of $B d$ (McKnight et al. 2019), none of the parks showed obvious signs of inbreeding or low diversity. Several factors affect a population's ability to retain diversity during an outbreak, including the duration of the decline, the number of individuals that survived the decline, and gene flow from neighboring populations (McKnight et al. 2017b). Thus, although diseases can cause a large loss of genetic diversity (Trudeau et al. 2004; Schoville et al. 2011; Albert et al. 2014; Serieys et al. 2015), many populations can endure a large population size reduction without experiencing bottlenecks or inbreeding (Morgan et al. 2008; le Gouar et al. 2009; Teacher et al. 2009; Lachish et al. 2011; Brüniche-Olsen et al. 2013). Populations of $L$. dayi at Wooroonooran and Tully appear to be robust, with high densities of individuals occurring over large areas (McKnight, pers. obs.). The species was less dense at Girramay-Kirrama, but still occurred over a large area. In addition, based on the high levels of connectivity we observed, it is likely that our study populations benefitted from gene flow from populations we did not sample. The large number of individuals surviving in the lowlands, combined with gene flow, would allow the retention of high levels of genetic diversity, despite the loss of all populations at sites above 300-400-m elevation (Lachish et al. 2011; Whiteley et al. 2015; McKnight et al. 2017a; McKnight et al. 2019).

The observed diversity values for $L$. dayi were similar to previously reported values for $L$. serrata and $L$. nannotis (McKnight et al. 2019). At Girramay-Kirrama, L. dayi had slightly lower genetic diversity values than $L$. nannotis and $L$. serrata, but comparing the species across all sites, $L$. dayi at Wooroonooran and Tully generally had slightly higher genetic diversity than did $L$. serrata or $L$. nannotis at Girramay-Kirrama (Fig. 4). Further, at all three parks, $L$. dayi generally had higher diversity than did either L. serrata or L. nannotis at Paluma Range National Park. These comparisons are admittedly strained due to the fact that, in some cases, different parks were sampled for different species. However, the fact that $L$. dayi showed no signs of inbreeding, and has not been able to recover even at parks with high diversity, while both $L$. serrata and $L$. nannotis recovered even at sites with low diversity, suggests that a lack of genetic diversity is not precluding $L$. dayi from adapting to coexist with $B d$ at upland sites.

Effective population sizes for $L$. dayi (7.9-7499.3; median $=40.3$ ) were largely comparable with previously reported values for $L$. nannotis $(18.4-1756.5$; median $=$ $62.9)$ and $L$. serrata $(10.3-676.1 ; \quad$ median $=212.9)$ (McKnight et al. 2019). Effective population sizes for $L$. dayi at Wooroonooran and Girramay-Kirrama were generally low, but effective population sizes were higher at Tully, particularly at site $\mathrm{T} 2$, which had the highest density of $L$. dayi (based on the transect distance required to sample 30 frogs: $150 \mathrm{~m}$ as opposed to $350-1540 \mathrm{~m}$; median = $560 \mathrm{~m}$ ); this site was also close to numerous other small creeks populated by $L$. dayi. The other sampling sites were comparatively more isolated. We also note that Tully generally had lower infection intensities and prevalences compared with the other sites. In addition, the low effective population size values at some sites may be partially a sampling artifact because female $L$. dayi lives in the forest where we could not sample it (Hodgkison and Hero 1999).

\section{Adaptation hypothesis}

Our results are consistent with the hypothesis that $L$. dayi is currently undergoing adaptation to $B d$. Previous research in other systems has documented that there is a heritable component to $B d$ infection risk (Palomar et al. 2016), and several studies have found evidence of $B d$ driving selection (Grogan et al. 2018; Voyles et al. 2018; Kosch et al. 2019). Our results are consistent with those studies. All three outlier tests identified the same 18 markers, and the subsequent PERMANOVA based on those markers confirmed that infected and uninfected frogs were genetically distinct, even after accounting for elevation and park. This suggests that selection for resistance to $B d$ infection is occurring at those loci.

In addition, the BLAST search matched several of the markers to genes with potential implications for $B d$ infections. For example, five markers matched genes related to skin function as either their best (3) or second-best (3) match (one matched for both). One of these (NCBI: XM_018268914.1; $e$ value $=1.4$ ) was a keratin-associated protein, which is potentially of interest because $B d$ colonizes keratinized surfaces, resulting in electrolyte 
imbalances that can lead to death (Berger et al. 1998; Campbell et al. 2012). Further, three of the matches ( $e$ values $=0.4-1.4$ ) were for a fucosidase alpha-L gene (FUCA1; NCBI: XM_018564520.1), which was also implicated in $B d$ infection dynamics in southern corroboree frogs (Pseudophryne corroboree, Kosch et al. 2019). That study used a laboratory infection trial followed by genotyping each frog, and it used both outlier detection methods and a genome-wide association analysis to look for genes that were associated with $B d$ tolerance. Of 29 SNPs that they identified as potentially related to $B d, 20$ matched fucosidase alpha- $\mathrm{L}$ genes. This agreement among studies is interesting, given that $L$. dayi and $P$. corroboree are not closely related. Nevertheless, there is good reason to suspect that fucosidase alpha-L genes would be involved in infection dynamics, because fucose is common on amphibian skin and likely plays a role in defense against pathogens, possibly by preventing them from binding to the skin (Meyer et al. 2007). In addition, in mammals, fucosidase plays a role in the formation of the stratum corneum, the layer predominantly infected by $B d$ (Nemanic et al. 1983; Berger et al. 1998; Abdayem et al. 2016).

Several other genes are also noteworthy. For example, two of our sequences matched genes related to immune function (NCBI: KY587143.1 and XM_018243675.1; $e$ value $=4.9$ and 1.4 , respectively), one of which (NCBI: KY587143.1) is associated with the MHC II complex. In addition, both our results and the results of Kosch et al. (2019) found an association between $B d$ and the same sodium bicarbonate solute carrier, family 4 (slc4a9; NCBI: XM_012959752.2; $e$ value $=4.9$ ). Finally, four of our SNPmatched genes involved in the regulation of tumors or apoptosis (two SNPs matched for both the best and secondbest match; $e$ value $=0.4-4.9$ ). Two of those genes were specifically related to tumor regulation in skin cells (NCBI: XM_018560900.1 and XM_018560908.1). Genes that regulate cell proliferation and apoptosis may have an important role in the progression of $B d$ infections (Richmond et al. 2009). Indeed, a study that compared gene expression in $B d$-sensitive and tolerant species, as well as between infected and uninfected individuals, found significant differences in a large number of genes related to apoptosis (Ellison et al. 2014). Another study (Brannelly et al. 2017) examined apoptosis in two species of experimentally infected frogs (including a Litoria species), and found that infection load correlated positively with cell death, further supporting the notion that apoptosis has an important role in infections.

The agreement among outlier detection methods, agreement with previous research, and biological plausibility of the genes identified, all support the hypothesis that $L$. dayi is currently adapting to $B d$ or, at the least, has genetic variation for traits that have important roles in $B d$ infections.
Nevertheless, several caveats should be acknowledged. First, the results of our BLAST searches were often lowquality matches, reducing confidence in them. Second, this study was entirely observational. Therefore, although our results are suggestive, more work on $L$. dayi is needed before we can confirm that it is adapting to $B d$. It would be particularly useful to employ techniques such as controlled heritability genetic parameter investigations and transcriptomics. In addition, these efforts are currently hindered by a shortage of genetic resources for frogs in the family Pelodryadidae (and "treefrogs" more generally), and a reference genome for a member of this group would greatly enhance our ability to test for adaptation to $B d$.

\section{Mechanisms allowing recolonization}

Our results suggest that $L$. dayi may be in the process of adapting to $B d$, despite a lack of upland recolonization. Therefore, it is worth briefly discussing how $L$. dayi could be adapting without experiencing upland population recoveries, as well as discussing other mechanisms that could allow recolonization. Although lowland populations of $L$. dayi have not experienced the massive declines that occurred in the uplands, our results show that many lowland individuals do become infected by $B d$, particularly at the highest elevation sites $(\sim 300-400 \mathrm{~m})$ where they currently occur. These infections likely have fitness costs, even when they are sublethal (Chatfield et al. 2013; Campbell et al. 2019), which would result in selective pressure. The strength of that selective pressure is not clear, however, and it could take many generations before alleles that confer resistance or tolerance to $B d$ are common enough in populations to allow the recolonization of upland sites (Robinson et al. 2012). Here again, more research should be conducted on this species to determine the strength of selection, and follow populations for several generations to attempt to document selection in action.

There are also several other potential explanations for the differential recovery patterns of Australia's rainforest frogs that were beyond the scope of this paper. For example, in some other systems, a shift in the timing of reproduction has allowed populations to recover from declines associated with $B d$ (Scheele et al. 2015). This has not been tested for our system, but it is possible that $L$. nannotis and $L$. serrata underwent such a shift, while $L$. dayi did not. In addition, differences in skin microbial communities or antimicrobial peptides may have played a role in the differential recovery patterns, as has been suggested in other systems (Kueneman et al. 2016; Jani et al. 2017; Bates et al. 2018; Bell et al. 2018). We are investigating this possibility, and our current results suggest that the skin microbiomes of $L$. dayi do differ from those of L. nannotis and L. serrata (McKnight 2019 [unpublished thesis]). 
These possibilities are not mutually exclusive with the hypothesis that adaptation to $B d$ is occurring. For example, fucose levels on the skin could affect the microbiome, which could, in turn, affect $B d$. Thus, adaptation may be driven by interactions between the microbiota and $B d$. Such pathways are clearly speculative, however, and future studies should continue to examine this system, test these possibilities, and further our understanding of the factors that allow some populations to recover while precluding recovery in others.

\section{Conclusion}

We tested three hypotheses for the lack of upland recolonization in $L$. dayi, and our results suggest that neither lowdispersal abilities nor a lack of genetic diversity can explain the absence of population recoveries. We did, however, find consistent evidence that some loci are undergoing selection. Thus, it is possible that $L$. dayi is currently in the process of adapting to resist infection by $B d$, but more research is needed to confirm this, ideally including controlled heritability trials.

\section{Data availability}

All data in this paper are available in the Supplementary Information. An identical copy is also available on Dryad (https://doi.org/10.5061/dryad.0gb5mkkz6).

Acknowledgements We are indebted to Eric Nordberg and Donna Simmons for their assistance collecting these samples. In addition, we would like to thank the members of the Molecular Ecology and Evolution lab for their advice and assistance with laboratory work. Finally, we would like to thank the Wet Tropics Management Authority and James Cook University College of Science and Engineering for funding this research. This work was conducted under Department of Environment and Heritage Protection permits \#WITK16243115 and \#WITK18531217, and with the approval of James Cook University's animal ethics committee (\#A2209 and \#A2437).

\section{Compliance with ethical standards}

Conflict of interest The authors declare that they have no conflict of interest.

Publisher's note Springer Nature remains neutral with regard to jurisdictional claims in published maps and institutional affiliations.

\section{References}

Abdayem R, Formanek F, Minondo AM, Potter A, Haftek M (2016) Cell surface glycans in the human stratum corneum: distribution and depth-related changes. Exp Dermatol 25:865-871

Albert EM, Fernández-Beaskoetxea S, Godoy JA, Tobler U, Schmidt BR, Bosch J (2014) Genetic management of an amphibian population after a chytridiomycosis outbreak. Conserv Genet $16: 103-111$
Bates KA, Clare FC, O’Hanlon S, Bosch J, Brookes L, Hopkins K et al. (2018) Amphibian chytridiomycosis outbreak dynamics are linked with host skin bacterial community structure. Nat Commun 9:1-11

Belkhir K (2004) Genetix 4.05.2. Lab Génome Popul, Univ Montpellier II, Montpellier, France

Bell SC, Garland S, Alford RA (2018) Increased numbers of culturable inhibitory bacterial taxa may mitigate the effects of Batrachochytrium dendrobatidis in Australian Wet Tropics frogs. Front Microbiol 9:1-14

Bell, SC, Heard GW, Berger L, Skerratt LF (2020) Connectivity over a disease risk gradient enables recovery of rainforest frogs. Ecol Appl https://doi.org/10.1002/eap.2152. Online ahead of print

Berger L, Speare R, Daszak P, Green DE, Cunningham AA, Goggin CL et al. (1998) Chytridiomycosis causes amphibian mortality associated with population declines in the rain forests of Australia and Central America. Proc Natl Acad Sci USA 95:9031-9036

Bolnick D, Caldera E, Matthews B (2008) Evidence for asymmetric migration load in a pair of ecologically divergent stickleback populations. Biol J Linn 94:273-287

Boyle DG, Boyle DB, Olsen V, Morgan JAT, Hyatt AD (2004) Rapid quantitative detection of chytridiomycosis (Batrachochytrium dendrobatidis) in amphibian samples using real-time Taqman PCR assayo Title. Dis Aquat Organ 60:141-148

Brannelly LA, Roberts AA, Skerratt LF, Berger L (2017) Epidermal cell death in frogs with chytridiomycosis (MÁ Esteban, Ed.). PeerJ 5:e2925

Brüniche-Olsen A, Burridge CP, Austin JJ, Jones ME (2013) Disease induced changes in gene flow patterns among Tasmanian devil populations. Biol Conserv 165:69-78

Campbell CR, Voyles J, Cook DI, Dinudom A (2012) Frog skin epithelium: electrolyte transport and chytridiomycosis. Int J Biochem Cell Biol 44:431-434

Campbell L, Bower DS, Clulow S, Stockwell M, Clulow J, Mahony M (2019) Interaction between temperature and sublethal infection with the amphibian chytrid fungus impacts a susceptible frog species. Sci Rep 9:83

Carvajal-Rodriguez A (2017) HacDivSel: two new methods (haplotype-based and outlier-based) for the detection of divergent selection in pairs of populations. PLoS ONE 12:e175944

Chatfield MWH, Brannelly LA, Robak MJ, Freeborn L, Lilvaux SP, Richards-Zawacki CL (2013) Fitness consequences of infection by Batrachochytrium dendrobatidis in northern leopard frogs Lithobates pipens. Ecohealth 10:90-98

Daszak P, Berger L, Cunningham AA, Hyatt AD, Green DE, Speare R (1999) Emerging infectious diseases and amphibian population declines. Emerg Infect Dis 5:735-48

Daszak P, Cunningham AA, Hyatt AD (2000) Emerging infectious diseases of wildlife-threats to biodiversity and human health. Science 287:443-449

Davies M, Richards SJ (1990) Developmental biology of the Australian hylid frog Nyctimystes dayi (Gunther). Trans R Soc South Aust 114:207-211

Do C, Waples RS, Peel D, Macbeth GM, Tillett BJ, Ovenden JR (2014) NeEstimator V2: re-implementation of software for the estimation of contemporary effective population size $(\mathrm{Ne})$ from genetic data. Mol Ecol Resour 14:209-214

Doyle JJ, Doyle JL (1987) A rapid procedure for DNA purification from small quantities of fresh leaf tissue. Phytochem Bull 19:11-15

Ellison AR, Tunstall T, Direnzo GV, Hughey MC, Rebollar EA, Belden LK et al. (2014) More than skin deep: functional genomic basis for resistance to amphibian chytridiomycosis. Genome Biol Evol 7:286-298

Excoffier L, Laval G, Schneider S (2005) Arlequin (version 3.0): an integrated software package for population genetics data analysis. Evol Bioinform Online 1:47-50 
Flanagan SP, Jones AG (2017) Constraints on the FST-heterozygosity outlier approach. J Hered 108:561-573

Foll M (2012) BayeScan v2.1 user manual. Ecology 20:1450-1462

Foll M, Gaggiotti O (2008) A genome-scan method to identify selected loci appropriate for both dominant and codominant markers: a Bayesian perspective. Genetics 180:977-993

Fox J, Weisberg S (2011) An R companion to applied regression, 2nd edn. Sage, CA

le Gouar PJ, Vallet D, David L, Bermejo M, Gatti S, Levréro F et al. (2009) How Ebola impacts genetics of western lowland gorilla populations. PLoS ONE 4:e8375

Grogan LF, Robert J, Berger L, Skerratt LF, Scheele BC, Castley JG et al. (2018) Review of the amphibian immune response to chytridiomycosis, and future directions. Front Immunol 9:1-20

Guarnizo CE, Cannatella DC (2013) Geographic determinants of gene flow in two sister species of tropical Andean frogs. J Hered 105:216-225

Hodgkison SC, Hero J-M (1999) Seasonal behaviour of Litoria nannotis, Litoria rheocola and Nyctimystes dayi in Tully Gorge, North Queensland, Australia. In: Frogs in the community. Proceedings of the Brisbane Symposium, p. 29-39, http://www. qldfrogs.asn.au/

Ingram GJ, McDonald KR (1993) An update on the decline of Queensland's frogs. In: Lunney D, Ayers D (eds) Herpetology in Australia: a diverse discipline, Zoological Society of New South Whales. Mosman, NSW, p. 297-303

Jani AJ, Knapp RA, Briggs CJ (2017) Epidemic and endemic pathogen dynamics correspond to distinct host population microbiomes at a landscape scale. Proc R Soc B Biol Sci 284:20170944

Jombart $\mathrm{T}$ (2008) adegenet: a $\mathrm{R}$ package for the multivariate analysis of genetic markers. Bioinformatics 24:1403-1405

Keenan K, McGinnity P, Cross TF, Crozier WW, Prodohl PA (2013) diveRsity: an $\mathrm{R}$ package for the estimation and exploration of population genetics parameters and their associated errors. Methods Ecol Evol 4:782-788

Kjeldsen SR, Raadsma HW, Leigh KA, Tobey JR, Phalen D, Krockenberger A et al. (2019) Genomic comparisons reveal biogeographic and anthropogenic impacts in the koala (Phascolarctos cinereus): a dietary-specialist species distributed across heterogeneous environments. Heredity 122:525-544

Kilian A, Wenzl P, Huttner E, Carling J, Xia L, Blois H et al. (2012) Diversity arrays technology: a generic genome profiling technology on open platforms. Methods Mol Biol 888:67-89

Kosch TA, Silva CNS, Brannelly LA, Roberts AA, Lau Q, Marantelli $\mathrm{G}$ et al. (2019) Genetic potential for disease resistance in critically endangered amphibians decimated by chytridiomycosis. Anim Conserv 22:238-250

Kueneman JG, Woodhams DC, Van Treuren W, Archer HM, Knight $\mathrm{R}$, Mckenzie VJ (2016) Inhibitory bacteria reduce fungi on early life stages of endangered Colorado boreal toads (Anaxyrus boreas). ISME J 10:934-944

Lachish S, Miller KJ, Storfer A, Goldizen AW, Jones ME (2011) Evidence that disease-induced population decline changes genetic structure and alters dispersal patterns in the Tasmanian devil. Heredity 106:172-182

Lal MM, Southgate PC, Jerry DR, Bosserelle C, Zenger KR (2017) Swept away: ocean currents and seascape features influence genetic structure across the $18,000 \mathrm{Km}$ Indo-Pacific distribution of a marine invertebrate, the black-lip pearl oyster Pinctada margaritifera. BMC Genom 18:66

Laurance WF, McDonald KR, Speare R (1996) Epidemic disease and the catastrophic decline of Australian rain forest frogs. Conserv Biol 10:406-413

Lind CE, Kilian A, Benzie JAH (2017) Development of diversity arrays technology markers as a tool for rapid genomic assessment in Nile tilapia, Oreochromis niloticus. Anim Genet 48:362-364
Lips KR, Brem F, Brenes R, Reeve JD, Alford RA, Voyles J et al. (2006) Emerging infectious disease and the loss of biodiversity in a Neotropical amphibian community. Proc Natl Acad Sci USA 103:3165-3170

McDonald KR, Alford RA (1999) A review of declining frogs in northern Queensland. In: Campbell A (ed.) Declines and disappearances of Australian frogs, envrionment Australia. Environment Australia, Canberra, pp. 14-22

McKnight D (2019) Life finds a way: the recovery of frog populations from a chytridiomycosis outbreak. PhD Thesis. James Cook University.

McKnight DT, Alford RA, Hoskin CJ, Schwarzkopf L, Greenspan SE, Zenger KR et al. (2017a) Fighting an uphill battle: the recovery of frogs in Australia's Wet Tropics. Ecology 98:3221-3223

McKnight DT, Schwarzkopf L, Alford RA, Bower DS, Zenger KR (2017b) Effects of emerging infectious diseases on host population genetics: a review. Conserv Genet 18:1235-1245

McKnight DT, Lal MM, Bower DS, Alford RA, Zenger KR (2019) The return of the frogs: the importance of habitat refugia in maintaining diversity during a disease outbreak. Mol Ecol 28:2731-2745

Meyer W, Seegers U, Schnapper A, Neuhaus H, Himstedt W, ToepferPetersen E (2007) Possible antimicrobial defense by free sugars on the epidermal surface of aquatic vertebrates. Aquat Biol 1:167-175

Morgan MJ, Hunter D, Pietsch R, Osborne W, Keogh JS (2008) Assessment of genetic diversity in the critically endangered Australian corroboree frogs, Pseudophryne corroboree and Pseudophryne pengilleyi, identifies four evolutionarily significant units for conservation. Mol Ecol 17:3448-3463

Nemanic MK, Whitehead JS, Elias PM (1983) Alterations in membrane sugars during epidermal differentiation: visualization with lectins and role of glycosidases. J Histochem Cytochem 31:887-897

Oksanen JF, Blanchet FG, Friendly M, Kindt R, Legendre P, McGlinn D et al. (2017) vegan: community ecology package.

Painter I (2013) GWASExactHW: exact Hardy-Weinburg testing for genome wide association studies. $\mathrm{R}$ package version 1.01.

Palomar G, Bosch J, Cano JM (2016) Heritability of Batrachochytrium dendrobatidis burden and its genetic correlation with development time in a population of common toad (Bufo spinosus). Evolution 70:2346-2356

Purcell S, Neale B, Todd-Brown K, Thomas L, Ferreira M, Bender D et al. (2007) PLINK: a toolset for whole-genome association and population-based linkage analysis. Am J Hum Genet 81:559-575

Richards SJ, Alford RA (2005) Structure and dynamics of a rainforest frog (Litoria genimaculata) population in northern Queensland. Aust J Zool 53:229-236

Richards SJ, McDonald KR, Alford RA (1993) Declines in populations of Australia's endemic tropical rainforest frogs. Pac Conserv Biol 1:66-77

Richmond JQ, Savage AE, Zamudio KR, Rosenblum EB (2009) Toward immunogenetic studies of amphibian chytridiomycosis: linking innate and acquired immunity. Bioscience 59:311-320

Robinson SJ, Samuel MD, Johnson CJ, Adams M, McKenzie DL (2012) Emerging prion disease drives host selection in a wildlife population. Ecol Appl 22:1050-1059

Sansaloni C, Petroli C, Jaccoud D, Carling J, Detering F, Grattapaglia D et al. (2011) Diversity arrays technology (DArT) and nextgeneration sequencing combined: genome-wide, high throughput, highly informative genotyping for molecular breeding of Eucalyptus. BMC Proc 5:P54

Scheele BC, Hunter DA, Skerratt LF, Brannelly LA, Driscoll DA (2015) Low impact of chytridiomycosis on frog recruitment enables persistence in refuges despite high adult mortality. Biol Conserv 182:36-43

Scheele BC, Pasmans F, Skerratt LF, Berger L, Martel A, Beukema W et al. (2019) Amphibian fungal panzootic causes catastrophic and ongoing loss of biodiversity. Science 363:1459 LP-1463 
Scheele BC, Skerratt LF, Grogan LF, Hunter DA, Clemann N, Mcfadden $\mathrm{M}$ et al. (2017) After the epidemic: ongoing declines, stabilizations and recoveries in amphibians afflicted by chytridiomycosis. Biol Conserv 206:37-46

Schoville SD, Tustall TS, Vredenburg VT, Backlin AR, Gallegos E, Wood DA et al. (2011) Conservation genetics of evolutionary lineages of the endangered mountain yellow-legged frog, Rana muscosa (Amphibia: Ranidae), in southern California. Biol Conserv 144:2031-2040

Serieys LEK, Lea A, Pollinger JP, Riley SPD, Wayne RK (2015) Disease and freeways drive genetic change in urban bobcat populations. Evol Appl 8:75-92

Sivasankaran RM, Purimetla B, Stankovic JA, Ramamritham K (1993) Network services database-a distributed active real-time database (dartdb) application. In: Proceedings of the IEEE Workshop on Real-Time Applications. IEEE, New York, p. 184-187

Smith KF, Sax DF, Lafferty KD (2006) Evidence for the role of infectious disease in species extinction and endangerment. Conserv Biol 20:1349-57

Steinig EJ, Neuditschko M, Khatkar MS, Raadsma HW, Zenger KR (2015) NetView P: a network visualization tool to unravel complex population structure using genome-wide SNPs. Mol Ecol Resour 16:216-227

Teacher AGF, Garner TWJ, Nichols RA (2009) Evidence for directional selection at a novel major histocompatibility class I marker in wild common frogs (Rana temporaria) exposed to a viral pathogen (Ranavirus). PLoS ONE 4:e4616

R Core Development Team (2017) R: A language and environment for statistical computing. R Core Development Team. Vienna, Austria

Trudeau KM, Britten HB, Restani M (2004) Sylvatic plague reduces genetic variability in black-tailed prairie dogs. J Wildl Dis 40:205-211

Voyles J, Woodhams DC, Saenz V, Byrne AQ, Perez R, Rios-sotelo G et al. (2018) Shifts in disease dynamics in a tropical amphibian assemblage are not due to pathogen attenuation. Sceince 359:1517-1519

Whiteley AR, Fitzpatrick SW, Funk WC, Tallmon DA (2015) Genetic rescue to the rescue. Trends Ecol Evol 30:42-49

Woodworth BL, Atkinson CT, LaPointe DA, Hart PJ, Spiegel CS, Tweed EJ et al. (2005) Host population persistence in the face of introduced vector-borne diseases: Hawaii amakihi and avian malaria. Proc Natl Acad Sci USA 102:1531-1536 